\title{
Duración absoluta de las vocales del español urbano y rural de la provincia de Nuble
}

\author{
Daniel Ignacio Pereira* \\ Jaime Soto Barba \\ Universidad de Concepción, Chile
}

\begin{abstract}
Resumen
El presente trabajo compara la duración absoluta de las vocales de tres grupos de hablantes del español de la provincia de Ñuble (Chile): urbano alto, urbano bajo y rural bajo. Los datos acústicos muestran que se manifiesta una relación entre estrato social y duración vocálica: mientras más cercano a la urbe y más alto sea el estrato social del hablante, menor será la duración de sus vocales y viceversa. Las explicaciones que pueden fundamentar este fenómeno están relacionadas con la mayor o menor tensión articulatoria, pero también con factores extralingüísticos como la concepción del tiempo de los informantes y la mayor o menor familiaridad con la situación comunicativa establecida para obtener las grabaciones.
\end{abstract}

Palabras clave: análisis acústico, duración vocálica absoluta, tensión articulatoria, habla urbana y rural.

\footnotetext{
* Para correspondencia, dirigirse a: Daniel Ignacio Pereira (danielpereira@udec.cl) o a Jaime Soto Barba (jstot@udec.cl), ambos del Departamento de Español, Facultad de Humanidades y Arte, Universidad de Concepción, Fono 2204313, Casilla 160-C, Correo 3, Concepción, Chile.
} 


\title{
Absolute length of urban and RuRal Spanish vowels of the \\ Nuble PROVINCE
}

\begin{abstract}
The present work compares the absolute length of three groups of speakers of Spanish of the Nuble province (Chile): urban upper middle class, urban lower class and rural lower class. The acoustic data shows a relationship between social status and vowels length. The closer to the urban centre and the higher the social class of the speakers the shorter their vowels and vice versa. The facts that can explain this phenomenon are related to more or less articulatory tension. However, it is also related to extra linguistic aspects such as the conception of time of the subjects, as well as the degree of familiarity with the communicative situation of the sample recording process.
\end{abstract}

Key words: Acoustic analysis, absolute vowel length, articulatory tension, urban and rural speech.

Recibido: 20/01/11. Aceptado: 09/05/11.

\section{INTRODUCCIÓN}

En un artículo anterior (Soto-Barba 2007), se presentó el comportamiento fonético acústico de los formantes vocálicos tónicos y átonos del español urbano y rural de la provincia de Ñuble. Entre los principales resultados destacaba el hecho de que los valores de los formantes vocálicos de los hablantes rurales eran más estables y se acercaban más a los patrones fonéticos-fonológicos esperados para el español (cf. Delattre 1962; Quilis 1981; Valdivieso y Tapia 1993) que los formantes vocálicos de los hablantes urbanos. En dicho artículo, una de las explicaciones posibles para este fenómeno consistía en la mayor duración absoluta de las vocales en los hablantes campesinos respecto de los urbanos.

En la lengua española, la mayor o menor duración de las vocales depende de la sílaba tónica o átona respectivamente, aunque no existen grandes diferencias entre ellas (cf. Martínez Celdrán 1996: 122). Es más, el parámetro de la duración no se relaciona con el carácter contrastivo de los sonidos en dicha lengua (cf. Quilis 1981), por lo que cabe suponer 
que las diferencias sistemáticas en la cantidad de tiempo invertido en la producción de un sonido vocálico, sobre todo cuando esta se da en algún estrato claramente diferenciado de la población, pudiera estar vinculada con variables pragmáticas, diastráticas o diatópicas, como parece ser el caso en la comparación entre las hablas rurales y urbanas de la provincia de Ñuble.

Muchos fonetistas consideran que la duración es uno de los índices de la tensión de los sonidos, no tanto porque la duración en sí misma produzca tensión, sino porque toda tensión conlleva forzosamente una mayor duración: la tensión exige un mayor esfuerzo muscular y para realizarlo se necesita más tiempo, ya que un gesto tenso exige por sí mismo un mayor tiempo de permanencia de los órganos en su posición que un gesto relajado (cf. Martínez Celdrán 1998: 53-54).

\section{PROCEDIMIENTOS Y MÉTODOS}

Para la recopilación de los datos se utilizaron entrevistas personales, realizadas en las residencias de los informantes, mediante un cuestionario que abordó diversos tópicos de la vida cotidiana y laboral de los sujetos. En total, se entrevistó a 12 informantes, cuatro de ellos de nivel sociocultural medio alto urbano, cuatro de nivel sociocultural bajo urbano y cuatro de nivel sociocultural bajo rural. El nivel sociocultural fue asociado al nivel educacional de los hablantes y la procedencia geográfica, de acuerdo al lugar de residencia habitual de los informantes.

El primer grupo (nivel sociocultural urbano alto) contiene sujetos con formación universitaria o terciaria completa, que viven en sectores acomodados del centro urbano de la ciudad más importante de la provincia de Ñuble, esto es, Chillán. Son todos profesionales que ejercen en instituciones públicas o privadas de acuerdo a su especialización.

El segundo grupo (nivel sociocultural urbano bajo) se compone de sujetos con formación secundaria o enseñanza media completa o incompleta, residentes en sectores periféricos de la ciudad. Se trata de obreros que desempeñan un oficio menor remunerado.

El tercer y último grupo (nivel sociocultural rural bajo) está integrado por hablantes con formación básica completa o incompleta, residentes en zonas rurales cercanas a la localidad de El Carmen, en la provincia de Ñuble. Son todos campesinos que trabajan su propia tierra o trabajan la de un dueño de fundo. 
La grabación de la muestra se realizó usando una grabadora de casete portátil Tascam, convencional, y con un micrófono Shure, modelo Prologue 14h-LC. Para un mejor y más eficiente manejo del corpus grabado originalmente en cintas magnéticas análogas, estas se digitalizaron posteriormente. Este proceso se realizó con el software Adobe Audition, en su versión 2.0. Además, fue necesario amplificar la señal y extraer el ruido originado por el tiempo de almacenamiento de las cintas. Este último paso se realizó con el plugin X-Noise, contenido en el software Waves 5.0.

El análisis acústico propiamente tal se realizó mediante el Espectrógrafo Digital 5500 de Kay Elemetrics, perteneciente al Laboratorio de Fonética de la Universidad de Concepción, con el programa Sonograma y Espectro de Energía.

El corpus analizado estuvo constituido por 400 vocales (100 por cada informante; 20 por cada una de las vocales). En la medición no se consideraron los marcadores discursivos orientados a no perder el turno de la palabra, constituidos acústicamente por sílabas con vocales notoriamente largas (cf. Soto-Barba y Roldán 2002).

\section{RESULTADOS}

\subsection{DuRACión ABSOLUTA DE VOCALES TÓNICAS}

La comparación de los valores de las vocales acentuadas que muestra la Tabla 1 permite señalar que la duración vocálica absoluta es mayor en los hablantes rurales, le siguen los hablantes urbanos del nivel sociocultural bajo y es menor en los hablantes urbanos de condición sociocultural alta.

Figura 1: Tabla y gráfico del promedio de la duración absoluta de las vocales tónicas, expresado en milisegundos

\begin{tabular}{|c|c|c|c|c|c|}
\hline INFORMANTES & $/ \mathbf{i} /$ & $/ \mathbf{e} /$ & $/ \mathbf{a} /$ & $/ \mathbf{o} /$ & $/ \mathbf{u} /$ \\
\hline Urbalto & 58 & 61 & 63 & 48 & 46 \\
\hline Urbajo & 69 & 68 & 75 & 82 & 80 \\
\hline Rurbajo & 86 & 97 & 98 & 94 & 93 \\
\hline
\end{tabular}




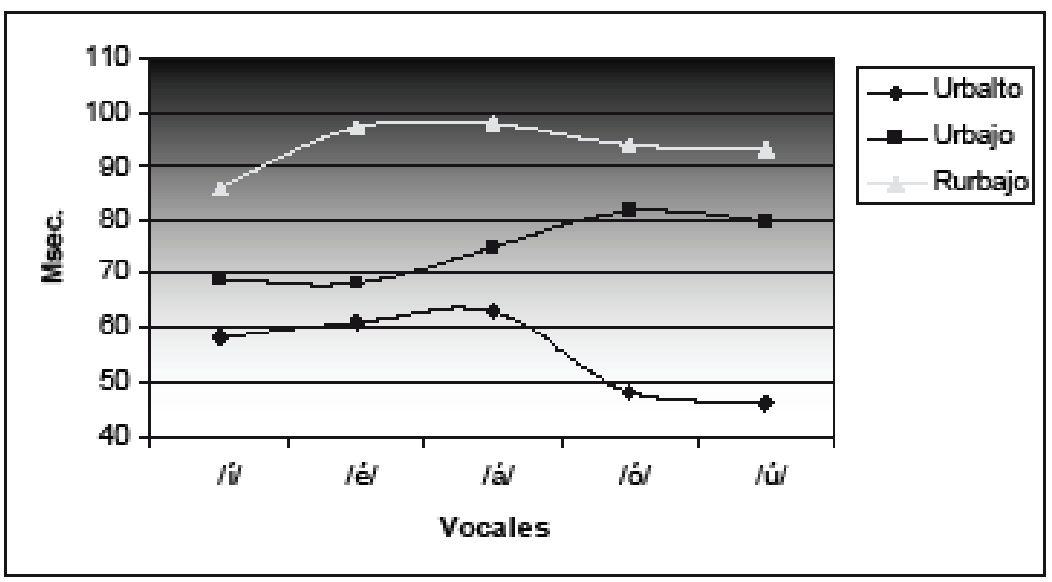

En el Gráfico 1 se muestra que todas las vocales presentan una mayor duración en los hablantes rurales de nivel sociocultural bajo (entre 86 y 98 milisegundos), le siguen los hablantes urbanos de condición sociocultural baja (entre 69 y 80 milisegundos) y la menor duración absoluta de los segmentos vocálicos corresponde a los hablantes urbanos de nivel sociocultural alto (entre 46 y 63 milisegundos). Obsérvese también que ninguno de los resultados obtenidos se traslapa, si consideramos los promedios de cada una de las vocales y los grupos definidos, es decir, los promedios más bajos rurales siempre son más altos que los urbanos de nivel sociocultural bajo, y los promedios más bajos de estos últimos informantes siempre son más altos que los de nivel sociocultural alto.

Lo expresado en el párrafo anterior es congruente con lo que afirma SotoBarba (2007), en relación con lo observado en el F1 y F2 de las vocales: nuevamente los resultados ordenan de manera escalonada los tres grupos de hablantes analizados. En otras palabras, si consideramos que se manifiesta un paralelo entre duración vocálica menor y relajamiento articulatorio, entonces existe un mayor relajamiento en los hablantes urbanos de nivel sociocultural alto, un tanto menos en los hablantes urbanos de condición sociocultural baja y mucho menos en los hablantes rurales de situación sociocultural baja.

Ahora bien, el gráfico anterior también permite determinar en forma clara que, si se consideran las vocales anteriores, la duración absoluta de los segmentos vocálicos es más cercana entre los hablantes urbanos, alejándose en los hablantes rurales. Si, en cambio, el foco de atención lo constituyen las vocales posteriores, se observa una situación contraria, pues son los hablantes de condición sociocultural baja, sean rurales o urbanos, quienes muestran resultados más cercanos, alejándose, en este caso, los hablantes 
de condición sociocultural alta. En el mismo sentido, la vocal central abierta /á/ es el segmento que muestra promedios más estables entre los tres grupos estudiados, lo que es coincidente con el trabajo de Marín (1994-1995) sobre la duración vocálica del español.

\subsection{Duración absoluta de las vocales Átonas}

En cuanto a la duración absoluta de los segmentos vocálicos átonos, la tendencia es similar a la manifestada por los segmentos tónicos, aunque existen algunas diferencias, las que pueden ser observadas en la tabla y el gráfico de la Figura 2.

Figura 2: Tabla y gráfico del promedio de la duración absoluta de las vocales átonas, expresado en milisegundos

\begin{tabular}{|c|c|c|c|c|c|}
\hline INFORMANTES & $/ \mathbf{i} /$ & $/ \mathbf{e} /$ & $/ \mathbf{a} /$ & $/ \mathbf{o} /$ & $/ \mathbf{u} /$ \\
\hline Urbalto & 55 & 68 & 57 & 45 & 52 \\
\hline Urbano & 56 & 62 & 72 & 78 & 73 \\
\hline Rurbajo & 69 & 68 & 74 & 81 & 85 \\
\hline
\end{tabular}

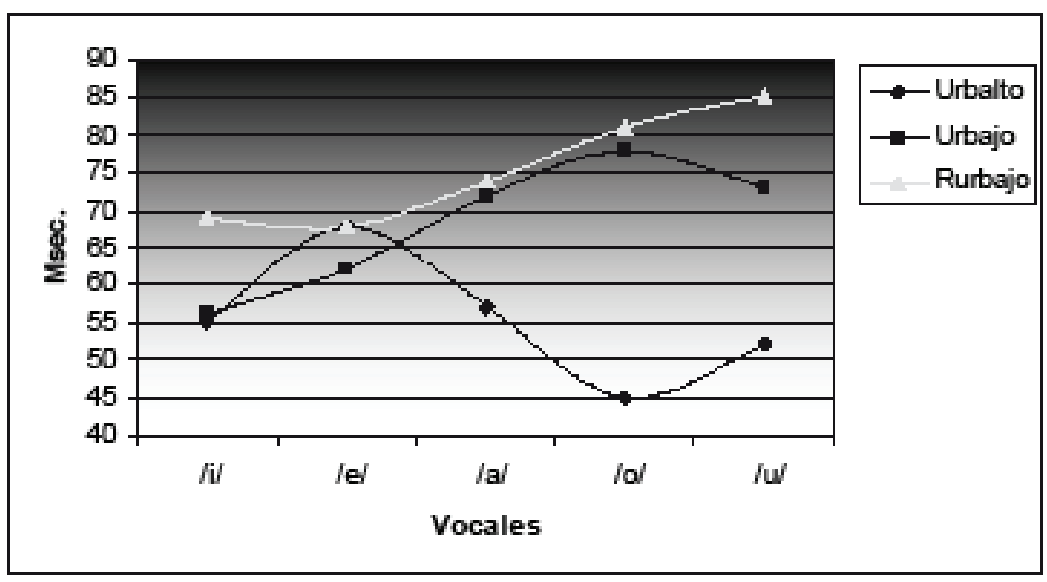

En efecto, los resultados presentados en la tabla y el gráfico de la Figura 2 indican que los hablantes rurales de condición sociocultural baja presentan promedios más altos que los otros dos grupos, es decir, producen vocales 
más largas; le siguen los hablantes urbanos de nivel sociocultural bajo, y nuevamente los hablantes urbanos de nivel sociocultural alto muestran vocales más cortas. La única vocal que no se ajusta a esta tendencia es la vocal /e/.

\section{CONCLUSIONES}

Como se pudo observar en los resultados presentados, es posible diferenciar los tres grupos de hablantes estudiados midiendo la duración absoluta de sus vocales tónicas y átonas. La duración de las vocales tónicas presenta una diferenciación total entre cada grupo considerado, y casi total en las vocales átonas, las que solo se traslapan en la vocal/e/. De este modo, los hablantes de condición sociocultural alta que viven en centros urbanos tienden a producir vocales más breves que las que producen hablantes urbanos de condición sociocultural baja y estos, a su vez, producen vocales más cortas que las que utilizan, en su actuación lingüística, los hablantes rurales de condición sociocultural baja. Por lo tanto, es posible establecer la siguiente relación en cuanto a la duración vocálica absoluta de estos hablantes:

\section{-duración +duración}

urbano alto < urbano bajo < rural bajo

La justificación que podría explicar este fenómeno no se orienta a la estructura lingüística sonora que caracteriza al español de Chile. Más bien, las razones pueden obedecer a comportamientos extralingüísticos de los grupos humanos definidos. Si se considera este planteamiento, es posible que la mayor o menor duración vocálica guarde relación con una percepción diferenciada del tiempo entre estos tres grupos humanos. De hecho, en los sectores rurales, se observa una forma de vida más pausada y tranquila, mientras que en la urbe, el estilo de vida es mucho más acelerado y rápido, lo que se acrecienta a medida que se asciende en el nivel sociocultural de los hablantes. En efecto, en lo que respecta a la sociedad chilena

Chile establece culturalmente una diferencia entre los ritmos de Santiago como gran urbe industrializada y acelerada y los sujetos de provincia con una vinculación más rural. Evidentemente en la urbanidad influyen 
aspectos propios de la sociedad industrial moderna (...), con una idea de escasez de tiempo. Por su parte, en la ruralidad persiste una actividad temporal cíclica, propia de la actividad productiva campesina donde cada cosa tiene su tiempo. El cultivo, la crianza y la explotación de los recursos naturales responden a temporadas que no se pueden alterar (Cladellas y Cárcamo 2009: 76).

Ciertamente, un campesino no establece una comunicación en la que sienta que no dispone del tiempo suficiente para interactuar con el interlocutor; su interacción lingüística, en consecuencia, es lenta y pausada. La situación con los hablantes urbanos, sobre todo los de nivel sociocultural alto, es totalmente opuesta: para un profesional-como los analizados en este estudio- el tiempo es extremadamente importante y puede sentir que una interacción lingüística constituye una pérdida de tiempo, por consiguiente, sus emisiones serán rápidas y sin pausas. Indudablemente, lo anterior implica emisiones sonoras más largas para el primer caso y más breves o cortas para el segundo, lo que naturalmente se expresa en las vocales. El hecho de que los hablantes urbanos de nivel sociocultural bajo se ubiquen, de acuerdo a los resultados, en una situación intermedia, refuerza la idea anterior, porque este grupo también pertenece a una clase sociocultural baja, pero se encuentra más cerca del centro urbano más importante de la provincia de Ñuble, como lo es la ciudad de Chillán.

Otra explicación posible consiste en que los hablantes rurales y de condición sociocultural baja se hayan sentido "evaluados" por el entrevistador, lo que podría haber incidido en su producción de habla, llevándolos a pensar mejor sus respuesta y pronunciarlas con lentitud para articular bien todos los sonidos. Esto explicaría la mayor tensión de las articulaciones vocálicas en los hablantes rurales, de acuerdo a Soto-Barba (2007). La presencia de tecnología, como los instrumentos de grabación, el micrófono, etc., podrían haber reforzado esta percepción de la situación comunicativa, situación que no habría ocurrido con los hablantes urbanos de condición alta, más acostumbrados a los aparatos tecnológicos que los rodean continuamente (cf. Morales 2005). Sin embargo, esta variación estilística de la duración vocálica podría ser estudiada en investigaciones posteriores. 


\section{REFERENCIAS BIBLIOGRÁFICAS}

Cladellas Pros, Ramón y Luis Ramón Cárcamo Ulloa. 2009. Percepción del tiempo de los estudiantes chilenos en tareas de Internet en función de su procedencia: urbana o semirural. SUMMA Psicológica UST 6, 2: 69-78.

Delattre, P. 1962.Le jeu des transitions de formants et perception des consonnes. $P 4^{\text {th }} I C P h S$, La Haya, Mouton : 407-417

Marín Gálvez, Rafael. 1994-1995. La duración vocálica del español. E.L.U.A. 10: 213 226.

Martínez Celdrán, Eugenio. 1996. El sonido en la comunicación humana. Barcelona: Ariel. 1998. Análisis espectrográfico de los sonidos del habla. Barcelona: Ariel.

Morales, Cesáreo. 2005. Tiempo psicológico en los estudiantes y carga de información. Ingenierías VIII, 27: 16-23.

Quilis, Antonio. 1981. Fonética acústica de la lengua española. Madrid: Gredos.

Soto-BARBA, JAIme y YASNA RoLdÁn. 2002. Marcadores discursivos en el habla urbana y rural de la provincia de Ñuble. Onomazein 7: 87-94.

Soto-BARbA, JAime. 2007. Variación del F1 y del F2 en las vocales del español urbano y rural de la provincia de Ñuble. $R L A$ 45, 2: 143-165.

VAldivieso, H. y M. TAPIA. 1993. Formantes vocálicos de un hablante representativo del español de Chile. Ponencia presentada en el "X Seminario de Investigación y Enseñanza de la Lingüística”. Universidad de Playa Ancha, Valparaíso, Chile. 